\title{
Estudio de la aplicación del método estándar del MARPOR para el posicionamiento ideológico de partidos argentinos, brasileños y chilenos en campañas presidenciales* \\ Study of the Application of the Standard Method of Marpor for \\ the Ideological Positioning of Argentine, Brazilian and Chilean Parties \\ in Presidential Campaigns
}

\section{FLÁVIO CONTRERA}

Universidade Estadual Paulista, Brasil

flavio.contrera@gmail.com

PRISCILLA LEINE CASSOTTA

Universidade Federal de São Carlos, Brasil

priscilla.leine@gmail.com

MATHEUS LUCAS HEBLING**

Universidade Estadual de Campinas, Brasil

matheushebling@gmail.com

https://doi.org/10.46468/rsaap.15.2.A8

\begin{abstract}
Resumen: La ampliación del alcance de aplicación del método de estimación ideológica del MARPOR para Sudamérica mostró posicionamientos de las plataformas partidarias argentinas, brasileñas y chilenas más a la izquierda de lo que generalmente son clasificadas por los especialistas. Con el objetivo de evaluar la adecuación del método estándar del MARPOR para estimar la posición ideológica de los manifiestos de estos países, este estudio concluyó que esta herramienta presenta imprecisiones en la construcción de la escala RILE. En el caso de los países sudamericanos, particularmente, estas imprecisiones se deben a la existencia de distorsiones causadas por el énfasis no partidista en relación con la ampliación del estado de bienestar social.
\end{abstract}

Palabras clave: América del Sur, MARPOR, Partidos Políticos, RILE

Abstract: The broadening of the scope of application of the MARPOR ideological estimation method for South America resulted in positioning of Argentine, Brazilian and Chilean partisan manifestos further to the left than what is generally classified by specialists. Objective to assess the adequacy of the MARPOR standard method to estimate the ideological position of the manifestos of these countries, this study concluded that this presents inaccuracies in the construction of the RILE scale. In the case of South American countries, particularly, these inaccuracies are due to the existence of distortions caused by the non-partisan emphasis in relation to the expansion of the social welfare state.

Keywords: South America, MARPOR, Political Parties, RILE

* Artículo recibido el 3 de septiembre de 2019 y aceptado para su publicación el 14 de julio de 2021.

** Los/as autores/as agradecen el aporte de evaluaciones anónimas a una versión previa de este artículo. 


\section{Introducción}

El eje izquierda-derecha es una medida importante para comprender distintos aspectos del proceso democrático, disponiendo de los partidos políticos como el centro de esta cuestión. Sin embargo, la clasificación ideológica de los partidos todavía es un desafío para politólogos que utilizan distintas variables para tal tarea (Tavits, 2007). Pese al uso recurrente de esa escala, es un problema práctico enfrentado por los estudiosos el encontrar medidas adecuadas para clasificar posicionamientos dentro de la dimensión izquierda-derecha (Gabel y Huber, 2000).

El Manifesto Research on Political Representation (MARPOR) -orginado en 1976 a partir de las contribuiciones de la saliency theory-, se ha empeñado en esa tarea tras el Manifesto Project. El MARPOR provee una medida para las posiciones de izquierda y de derecha, compuesta por una escala de variables sociales y económicas, conocida como RILE (Right-Left). Investigadores dedicados al estudio de los partidos y sistemas partidarios europeos reconocen que el RILE es uno de los mejores indicadores de tendencias sobre programas políticos (Budge, 2013; Volkens et al, 2015).

El objetivo de este artículo es evaluar la adecuación del método estándar del MARPOR para estimar la posición ideológica de los manifiestos de partidos/coaliciones argentinos, brasileños y chilenos en sus elecciones presidenciales. Para eso, analizamos los resultados de las estimaciones realizadas por los investigadores del MARPOR de las posiciones ideológicas de los manifiestos de campaña brasileños en las elecciones presidenciales de 1994, 1998, 2002, 2006, 2010 y 2014; de los argentinos en las elecciones presidenciales de 1995, 1999, 2003, 2007 y 2011; y de los chilenos en las elecciones presidenciales de 1989, 1993, 1999, 2005, 2009y $2013^{1}$. Nuestras inferencias se basaron en el contraste entre esas estimaciones ideológicas y aquellas realizadas por especialistas para los mismos partidos/coaliciones de aquellos países. La comparación de distintas clasificaciones ideológicas se emplea como estrategia metodológica para discutir la validez de esas clasificaciones (Tarouco y Madeira, 2013) ${ }^{2}$.

Aunque uno de los propósitos del MARPOR es comparar la posición ideológica entre los partidos en distintos países, hay una falta de adecuación cuando observamos la realidad sudamericana. Se crea un problema de validez, que este trabajo cree que puede resolverse a través de una construcción

En el caso de los tres países, los investigadores del MARPOR codificaron solamente los manifiestos de los partidos o coaliciones más votados en aquellas elecciones.

2 Los datos para replicar los resultados del presente estudio se encuentran disponibles en: http://ieee-dataport.org/4493 
de escala más informada teóricamente. Al respecto de esto, Mella et al (2019) consideran que existe una mayor complejidad en la definición de los conceptos de izquierda y derecha en América Latina, dada la relativa poca institucionalización de varios sistemas de partidos, así como también la existencia de variables subyacentes, tales como cierto grado de conservadurismo transversal a los actores políticos.

Acerca de la extensión de la aplicación de la RILE en Latinoamérica, autores que analizaron los casos de Argentina (D’Alessandro, 2013), Brasil (Tarouco y Madeira, 2013; Tarouco y Madeira, 2013) y Chile (Mella et al, 2019) y del Cono Sur como un todo (López et al, 2013), argumentan que hay problemas en la consideración de categorías como "Derechos Humanos" y "Constitucionalismo: Positivo" en el eje de la derecha. Como resultado del terrorismo cometido por los regímenes militares de derecha, la defensa de los Derechos Humanos y del constitucionalismo se encontraría asociada a la izquierda en esos países. Mella et al (2019) y López et al (2013) consideran también controvertida la inclusión de la categoría "Autoridad Política" en el eje de la derecha en América Latina, ya que esto sería una característica de los partidos de izquierda y derecha en el continente. Otra dificultad residiría en adaptar las especificidades históricas y socio-económicas del contexto europeo al escenario sudamericano (Tarouco y Madeira, 2013).

Para preservar la comparabilidad con los casos europeos, Mella et al (2019), D’Alessandro (2013) y López et al (2013) no han sugerido adaptaciones de la escala RILE para América Latina. A pesar de eso, López et al (2013) advierten que la aplicación íntegra del método estándar a los países latinoamericanos debe tener en cuenta eventuales distorsiones a la hora de interpretar los resultados. Así, sugieren que en el caso latinoamericano el análisis del eje izquierda-derecha debe combinarse con el estudio del eje economía-mercado y con la dimensión justicia social y Estado de Bienestar. Por otro lado, Tarouco y Madeira (2013), propusieron una escala adaptada a la realidad brasileña.

En el último decenio, un número creciente de trabajos ha discutido la extensión (Ares y Volkens, 2017) y la aplicación del método estándar del MARPOR en América Latina ${ }^{3}$. Sin embargo, cuando MARPOR presentó en su base de datos (Lehmann et al, 2018) los resultados de la codificación de los manifiestos de Argentina, Brasil y Chile, constatamos que estos casos

Además de los trabajos ya citados, destacamos las contribuciones de Ares y Pérez (2017) para el caso argentino; Tarouco (2011), Tarouco y Madeira (2015); Cervi y Gandin (2017) para el caso brasileño; Gamboa et al (2013), Ruiz y Miranda (2017), Madariaga y Kaltwasser (2019) para el caso chileno; Martínez-Hernández y Martínez (2017) para el caso mexicano; Guedes et al (2011), Lorenzoni y Pérez (2013) para el caso uruguayo. 
fueron posicionados en la RILE más a la izquierda de lo que generalmente son clasificados por expertos de estos países. Desde esa constatación, nuestra hipótesis es que algunas categorías de la RILE perjudican la estimación de la posición real de los partidos de esos países. Eso ocurriría porque las categorías como "Expansión del Estado de bienestar" y "Expansión de la Educación" serían incorporadas por los partidos políticos, en el contexto sudamericano, de manera muy distinta del contexto europeo. Concluimos que el método de estimación ideológica del MARPOR presenta imprecisiones en la construcción de la escala RILE debido a la manera en que es incorporada a esta la saliency theory; también en relación al método de medición de las frecuencias de categorías; y en el caso de los países sudamericanos, particularmente, a la existencia de distorsiones derivadas del consenso acerca de la ampliación del bienestar social.

Este estudio está organizado en cinco secciones. Después de esta introducción discutimos, en el segundo apartado, el impacto de la saliency theory en la construcción de la RILE. En la tercera parte, evaluamos las imprecisiones de medición de las categorías de la RILE a partir de la identificación de las perspectivas teóricas que fundamentan la creación de aquellas categorías. En la cuarta sección discutimos los resultados de la aplicación de la RILE en el contexto de Brasil, Chile y Argentina, aclarando la cuestión de la defensa del estado de bienestar social. Finalmente, en la quinta sección, presentamos las conclusiones.

\section{2. ¿La perspectiva de la saliency theory dificulta la construcción de la escala RILE?}

Las teorías de la competencia entre partidos explican que estos últimos se posicionan centrípetamente cuando responden a los llamados de la mayoría moderada de la opinión pública (Downs, 1957) y centrífugamente cuando responden a los llamados de sus militantes (Aldrich, 2011). En el primer caso, prevalece la convergencia de posiciones, en el segundo, la polarización. La saliency theory difiere de estas dos concepciones. Su argumento central es que la competencia partidaria se centraría en los énfasis selectivos en lugar de la confrontación ideológica (Robertson, 1976). Más que oponerse a las declaraciones de sus oponentes sobre las políticas defendidas, los partidos políticos centran su atención en temas en los que consideran tener ventajas sobre sus competidores.

Otro concepto importante en la saliency theory es el de issue ownership, entendido a través de la idea de que los votantes perciben a ciertos partidos 
como más competentes que sus oponentes en un área de política específica (Budge, 1982). De esta manera, los partidos políticos tienden a priorizar ciertos temas durante las elecciones, mientras que sus actitudes políticas pueden cambiar lentamente (Petrocik, 1996).

Desde la perspectiva de la saliency theory para fundamentar su método estándar, el principal objetivo del MARPOR pasó a ser la identificación de los asuntos más frecuentes del manifiesto de un partido, es decir, aquellos que los partidos ponen más atención. Esa es la principal estrategia del grupo para identificar las diferencias entre los partidos. Efectivamente, el cuadro analítico del MARPOR cuenta con 57 categorías, que, por cubrir un variado abanico de temáticas, auxilian el objetivo de identificar aquellas más frecuentes en cada manifiesto.

La escala RILE, a su vez, apunta a posicionar el manifiesto de un partido en un espacio ideológico y unidimensional que varía de la izquierda a la derecha. En ese sentido, el principal objetivo de esa escala es destacar las diferencias de posicionamiento ideológico entre los partidos. Sin embargo, la manera como esta escala fue construida parece limitar, al menos parcialmente, este objetivo. Antes de aclarar esta cuestión veamos en la Tabla 1 las 26 categorías de la RILE:

\section{TABLA 1}

Categorías de la Escala Izquierda-Derecha (RILE)

\begin{tabular}{clcl}
\hline Izquierda & Derecha & \\
\hline Código & Categoría & Código & Categoría \\
\hline per103 & Antiimperialismo & per104 & Ejército: Positivo \\
per105 & Ejército: Negativo & per201 & Libertad y Derechos Humanos \\
per106 & Paz & per203 & Constitucionalismo: Positivo \\
per107 & Internacionalismo: Positivo & per305 & Autoridad Política \\
per202 & Democracia & per401 & Libre empresa \\
per403 & Regulación del mercado & per402 & Incentivos \\
per404 & Planificación económica & per407 & Proteccionismo: Negativo \\
per406 & Proteccionismo: Positivo & per414 & Ortodoxia Económica \\
& & per505 & Restricción del Estado de \\
per412 & Economía controlada & bienestar \\
per413 & Nacionalización & per601 & Forma de vida nacional: Positivo \\
per504 & Expansión del Estado de bienestar & per603 & Moralidad Tradicional: Positivo \\
per506 & Expansión de la Educación & per605 & Ley y Orden Público \\
per701 & Grupos laborales: Positivo & per606 & Armonía Social \\
\hline
\end{tabular}

Fuente: Ares y Volkens (2017). 
Tal como es evidenciado en la Tabla 1, la principal estrategia del MARPOR consistió en agrupar en los espectros que ellos identifican como izquierda y derecha categorías que serían más salientes para partidos de izquierda y de derecha y no necesariamente categorías que expresan divergencias de posiciones sobre un mismo tema. Así, las categorías de izquierda se concentraron en temáticas de relaciones exteriores y en temáticas socio-económicas, mientras que las categorías de derecha se concentraron menos en relaciones exteriores, y más en temas que abordan el sistema político, los valores sociales y la economía. Solamente tres entre los trece pares de categorías están en oposición directa: "Ejército", "Proteccionismo" y "Expansión del Estado de bienestar".

Sin embargo, hay dentro del cuadro de 57 categorías de otras posibilidades de apareamiento no incluidas en esta escala, lo que resulta en una dificultad de comparar posiciones antagónicas sobre un mismo tema en la escala izquierda y derecha. En alguna medida, la opción de combinar elementos salientes de la diversidad ideológica tiene como efecto imprecisiones en la manera de medir las posiciones de los manifiestos de los partidos en la escala. Esa ambigüedad afecta no solo los partidos de los países sudamericanos, sino también los partidos de todos los países donde se aplica el método. Existe todavía una imprecisión anterior en la construcción de la escala RILE relacionada con la fundamentación teórica de las categorías. Analizaremos esta cuestión en la sección siguiente.

\section{Desajuste entre teoría y medición ideológica. Las categorías de izquierda de la escala RILE}

El método estándar de MARPOR puede entenderse como un proceso de dos pasos: identificar unidades y codificarlas. Cada parte textual del cuerpo de un manifiesto necesita ser identificada y codificada. Sin embargo, algunas partes del manifiesto no deben considerarse cuerpo del texto (por ejemplo, encabezamientos de capítulos y secciones, estadísticas, tablas de contenido, observaciones introductorias), sino que deben ignorarse (Werner et al., 2015).

La unidad de codificación del método del MARPOR es la quasi-sentence. Esta es definida como un argumento que es la expresión verbal de un tema o de una idea política. Está frecuentemente separada en el texto por coma o punto y coma. Las quasi-sentences que son identificadas en un manifiesto pasan por un proceso de codificación, donde individualmente son clasificadas en una de las 57 categorías del cuadro analítico del MARPOR (Klingemann et al, 2006). 
A menudo los actores políticos son muy claros en sus declaraciones y dicen con sinceridad lo que buscan. En este caso, asignar códigos es sencillo. Sin embargo, hay momentos en que estas declaraciones no son muy claras y son más difíciles de codificar. Por ejemplo, cuando ninguna de las categorías de la tabla se aplica a una quasi-sentence determinada, debe tratarse como no codificable (per000). Otro problema que puede surgir es que la quasi-sentence contiene argumentos que permiten clasificarla en más de una categoría. En este caso, el codificador debe decidir cuál es la preocupación más importante del argumento y clasificar la quasi-sentence en una sola categoría. Cuando algunas oraciones todavía parecen oscuras, el investigador debe tener en cuenta el contexto en el que se insertan y también las siguientes frases, ya que en ellas el argumento anterior puede explicarse mejor.

Una vez finalizado el proceso de codificación, se realiza el conteo de la frecuencia de las categorías temáticas. Todas las quasi-sentences clasificadas son contadas de la misma manera. La frecuencia final de cada categoría varía en cuanto a la métrica de cada manifiesto. Es decir, considerando que las métricas de los programas partidarios varían considerablemente, son calculados los porcentuales que cada categoría representa en relación al número total de sentencias en un programa.

Aunque el procedimiento de conteo de frecuencia de las categorías basado en la métrica del manifiesto sea adecuado, una imprecisión parece ocurrir en el momento anterior al conteo. Se trata del momento en el que es atribuido el peso a cada quasi-sentence clasificada individualmente. Todas las quasi-sentences son contadas de la misma manera, recibiendo peso 1 a cada vez en que surgen, lo que en un primer momento suena como un procedimiento adecuado. Sin embargo, una mirada más atenta puede revelar un profundo desajuste entre las teorías que fundamentan la construcción de las categorías y la métrica atribuida a las quasi-sentences clasificadas. Analicemos esta cuestión con mayor detenimiento.

Después de medir las frecuencias de las 57 categorías del MARPOR en cada programa partidario, se calcula la posición del partido emisor del manifiesto en una escala de izquierda y de derecha (RILE). Esa escala está compuesta por 26 categorías, 13 de las cuales representan idearios de izquierda y otras 13 representan idearios de derecha, conforme la Tabla 1. Para calcular el posicionamiento de los manifiestos en la RILE es necesario sustraer la suma de los porcentuales de las 13 categorías de izquierda de la suma de los porcentuales de las 13 categorías de derecha:

RILE: $\sum$ Right categories - $\sum$ Left categories 
Con el resultado de este cálculo es posible posicionar el manifiesto de un partido/coalición en una escala que varía de -100 hasta +100 , siendo esos puntos considerados como los extremos de izquierda y de derecha, respectivamente. Los investigadores del MARPOR no explican detalladamente por qué aquellas 26 categorías fueron elegidas entre los 57 totales. A pesar de esto, Budge (2013) reconoce la naturaleza deductiva ${ }^{4}$ del método $a$ priori y explica que las categorías ideológicas, tanto a la izquierda como a la derecha, se basan en escritos teóricos de principios del siglo XX. En el caso de las categorías de izquierda, los autores argumentan que ellas encuentran su fundamento teórico en los escritores marxistas que enfatizan la intervención estatal en la economía y el bienestar social, juntamente con la idea leninista de que la internacionalización del capitalismo por medio de la expansión colonial de las potencias imperialistas llevaría al conflicto y a la guerra. La oposición popular al capitalismo implicaba presión para reformas sociales internas y para una política exterior progresista y pacífica que sofocaría el modo final de supervivencia del capitalismo (Klingemann et al, 2006; Budge, 2013).

Sin embargo, al analizar las 13 categorías identificadas como izquierda, la primera constatación que podemos hacer que no todas encuentran fundamento teórico en los escritores marxistas. Es decir, hay categorías de izquierda fundamentadas en el keynesianismo y en una miríada de perspectivas liberales. La segunda, es que entre aquellas fundamentadas en el marxismo hay enormes diferencias de posicionamiento teórico, que separan, por ejemplo, marxistas ortodoxos de marxistas reformistas. Veamos atentamente esas diferencias, en cada uno de los dominios temáticos del MARPOR.

Las cuatro primeras categorías de izquierda pertenecen, en el cuadro analítico del MARPOR, al dominio político de las Relaciones Exteriores. Se tratan de las categorías "Antiimperialismo", "Ejército: negativo", "Paz" e "Internacionalismo: positivo". Analizadas en conjunto, esas categorías sugieren que una visión de izquierda de las relaciones exteriores sería medida por el no intervencionismo, por la reducción o mantenimiento de las fuerzas armadas al mínimo necesario y no utilizar de esas fuerzas para resolver conflictos, por la búsqueda de la paz y por la cooperación por vía de las

\footnotetext{
En contraste, las medidas inductivas han sido propuestas como alternativas al método estándar basado en el análisis factorial tanto por los investigadores de MARPOR (Budge, 1987; Laver y Budge, 1992) como por otros académicos (Gabel y Huber, 2000). La mayoría de las escalas inductivas están justificadas en términos de su mayor sensibilidad y precisión. Sus defensores consideran ofrecer medidas más precisas de las posiciones de los partidos políticos para un análisis comparativo. Válidas para los datos de las que surgen, las medidas inductivas son limitadas en sus aplicaciones más allá de estas (Budge, 2013).
} 
instituciones internacionales. En el cuadro teórico de las relaciones internacionales esa visión puede ser caracterizada de dos maneras. Resaltando el no intervencionismo y la crítica a todas las formas de imperialismo tenemos una visión liberal-aislacionista. De otra manera, resaltando las definiciones de las otras tres categorías tenemos una visión liberal-internacionalista.

Con respecto a esto, es más clara la contribución de la teoría marxista en relación con la categoría "Antiimperialismo". El MARPOR define el alcance de esta categoría en la identificación, en los manifiestos partidarios, de referencias negativas al comportamiento imperial o en la manifestación de fuerte influencia, sea política, militar o económica, de un Estado sobre otros Estados y también de organizaciones financieras internacionales sobre Estados. Esa categoría encuentra fundamento en la obra de Vladimir Lenin. Para Lenin (2010), la internacionalización del capitalismo por medio de la expansión colonial de las potencias imperialistas llevaría al conflicto y a la guerra.

De otro modo, las categorías "Ejército: negativo", "Paz" e "Internacionalismo: positivo" no son tributarias del marxismo. Sabemos que hay una perspectiva internacionalista dentro de la teoría, pero ella no influenció la definición de aquella categoría del MARPOR. En Marx y Engels (1963), el internacionalismo es definido como la estrategia del proletariado que debería ser concebido como una clase internacional. El autor creía que la lucha de clases se internacionalizaría en la medida en que la explotación del trabajo se convertiría en un problema social involucrando a todos los países que existen en la sociedad moderna. En ese sentido, la creación de una Asociación Internacional de los Trabajadores sería fundamental para que el interés común de los proletarios de todas las naciones fuera formulado y traducido en estrategias políticas.

Sin embargo, en el cuadro del MARPOR, "Internacionalismo: positivo" trata de la necesidad de cooperación entre Estados, apoyo a las organizaciones internacionales y a las estructuras de gobernanza global. Junto a "Ejército: negativo" y "Paz", la definición de "Internacionalismo: positivo" fue fuertemente influenciada por la tradición liberal de las relaciones internacionales. Frente a la naturaleza conflictiva derivada de la anarquía en el sistema internacional, los liberales vislumbran la posibilidad de cambiarla por un orden más cooperativo, posibilitado por medio de instituciones internacionales, cuya visión surge, sobre todo, de los escritos de Immanuel Kant (2003). Efectivamente, se rechaza el empleo de la violencia, como el uso de las fuerzas armadas, para la resolución de conflictos derivados de la anarquía.

A su vez, dentro del dominio político de la Economía están, en el cuadro del MARPOR, las categorías "Regulación del mercado", "Economía Controlada", "Planificación Económica", "Proteccionismo: Positivo" y "Na- 
cionalización”. Aunque todas esas categorías puedan ser asociadas a la izquierda, ellas no se fundamentan en el marxismo.

La categoría "Regulación del mercado" se refiere al apoyo a las políticas destinadas a crear un mercado económico justo y abierto. Hay diversas teorías que fundamentan la regulación. La teoría del interés público sostiene que la regulación es provista como respuesta a la demanda del público por la corrección de prácticas de mercado ineficientes o injustas. Bajo la perspectiva de Chicago, la regulación, en regla, es adquirida por la industria, proyectada y operada principalmente para su beneficio (Stigler, 1971). Por su lado, los teóricos de la elección pública de Virginia critican a los teóricos de Chicago por su desconsideración de las ineficiencias de la regulación. En contrapartida, los marxistas entienden que la regulación económica no se trata de interés público, si no que se trata de un proceso por el cual grupos de interés buscan promocionar sus intereses (privados), asumiendo varias formas distintas (Posner, 1974).

En la contribución de Rosa Luxemburgo (1954) podemos encontrar que los fundamentos de las categorías "Economía Controlada" -que tratan del control directo del gobierno sobre la economía, incluyendo control sobre los precios, introducción de sueldo mínimo y "Planificación Económica" se refieren a planes a largo plazo, de políticas y estrategias. Se trata de un mecanismo de asignación de recursos que es contrastado con el mecanismo de mercado. La estrategia de la planificación llevada a cabo por una economía controlada se tornó práctica corriente en las economías socialistas.

Por su parte, la categoría "Proteccionismo: positivo" es definida por el MARPOR considerando menciones favorables sobre la extensión o mantenimiento de medidas de protección de los mercados internos. En su teoría del proteccionismo general, Manoilescu (1929) defendía que los mecanismos de protección deberían concentrarse en sectores de rentabilidad crecientes y no solamente en sectores de productividad. Recientemente, autores argumentaron que la teoría de Manoislescu recuperó algunas de las ideas básicas de la teoría del valor de Marx, especialmente las interpretaciones postmarxistas del valor del trabajo y de la plusvalía generada en la moneda extranjera (Nenovsky y Torre, 2015). Sin embargo, aunque haya similitudes teóricas y que el proteccionismo contemporáneo sea defendido por partidos y gobiernos de izquierda, esa contribución no está teóricamente ni históricamente asociada al marxismo, ya que su origen se remonta al mercantilismo de Friedrich List.

El MARPOR entiende como "Nacionalización" menciones favorables respecto de la propiedad gubernamental de industrias, sean parciales o completas; y que defienden el mantenimiento de industrias nacionalizadas o a 
la estatización de industrias privadas. Es importante destacar, sin embargo, que la idea de nacionalización en sí misma nada tiene que ver con el socialismo y existió bajo regímenes no-socialistas e, incluso, anti socialistas (Hastings et al, 2000). En cambio, la nacionalización fue uno de los principales mecanismos defendidos por los socialistas reformistas y socialdemócratas para la transición gradual hacia el socialismo. En ese sentido, no es posible establecer una asociación directa entre marxismo ortodoxo y nacionalización.

Dentro del alcance del dominio "Bienestar y Calidad de Vida" el MARPOR seleccionó dos categorías representando a la izquierda. Se trata de las categorías "Expansión del Estado de bienestar" y "Expansión de la Educación", que encuentran fundamento teórico en el keynesianismo (Keynes, 1936).

En el dominio de los "Grupos Sociales", la categoría "Grupos Laborales: Positivo" se refiere, según el MARPOR, a cuestiones laborales y al apoyo a todos los grupos de trabajadores, sindicatos y a la clase trabajadora en general, incluyendo desempleados. En ese sentido, es clara la vinculación de esa perspectiva con la teoría marxista (Marx y Engels, 1963). Aunque la visión de Marx y Engels sobre organizaciones laborales se haya complejizado a lo largo del tiempo, se destaca que inicialmente que ellos las han entendido como siendo potencialmente revolucionarias (Botz, 2013).

Finalmente, aparece la categoría "Democracia", vinculada a la izquierda por MARPOR. Según Bobbio (1996), Democracia no podría ser clasificada ni como una categoría de izquierda ni como una categoría de derecha. Tomando el concepto de libertad, que es considerado un bien individual, el autor argumenta que el ideal de la libertad encuentra su realización en los principios y en las reglas que están en la base de los gobiernos democráticos. De otro modo, la radicalidad de proyectos de transformación, sean revolucionarios o contrarrevolucionarios, tienen en común la convicción de que su realización depende de la instauración de regímenes autoritarios. Así, la díada libertad y autoridad serviría sólo para distinguir el ala moderada del ala extremista, tanto en la izquierda como en la derecha. Esa concepción es compartida por Jahn (2010), para quien la referencia a la democracia no se inclina ni para la izquierda ni para la derecha, sino que, en cambio, es una medida para entender el grado de radicalismo.

Efectivamente, las categorías de izquierda del MARPOR encuentran su fundamento en distintas perspectivas teóricas. ¿De qué manera esas diferencias impactan en la construcción de la RILE? Entendemos que en este aspecto reside una imprecisión importante. Cuando equivalen categorías de izquierda como "Antiimperialismo" y "Expansión del Estado de bienestar", por ejemplo, se niegan las diferencias teóricas entre ellas. Entender 
esas diferencias es fundamental en la medida en que ellas moldean las posiciones y la identidad política de un partido. Al homogeneizar la manera del conteo de las categorías, el MARPOR no tiene en cuenta esas diferencias.

El resultado es que, hipotéticamente, si un partido de extrema izquierda defiende solamente posiciones en consonancia con el "Antiimperialismo" y un partido socialdemócrata se posiciona a favor de la "Expansión del Estado de bienestar", los dos estarán posicionados juntos al final, en la escala RILE, en la extrema izquierda, en la posición -100. Esto sucede aunque sea evidente que el partido que defiende solamente posiciones antiimperialistas estaría más a la izquierda (en el sentido teórico) que el partido que adopta apenas posiciones en favor de la expansión del Estado de bienestar. De este modo, la métrica de posicionamiento ideológico del MARPOR no corresponde a las diferencias teóricas que fundamentan las posiciones partidarias.

Esto se trata de una preocupación puramente deductiva. Las medidas inductivas alternativas al método estándar, como el método 'vanilla' (Gabel y Huber, 2000), son todavía más problemáticas desde nuestro punto de vista porque son ateóricas. Sin embargo, no pretendemos reproducir aquí un análisis comparativo del método estándar en relación con otras medidas alternativas, ya sean deductivas o inductivas, como ya han hecho Dinas y Gemenis (2009) y Budge (2013) $)^{5}$. En este sentido, nos limitamos sólo a destacar las imprecisiones que fundamentan las categorías de izquierda. También es evidente que no queremos afirmar, a partir de ese debate, que todos los autores aquí referenciados fueron los utilizados por los fundadores de MARPOR para construir esas categorías, lo que podría ser considerado especulativo.

Por el contrario, nuestro propósito ha sido discutir la eficacia del empleo de una regla de conteo homogénea para categorías construidas a partir de perspectivas teóricas heterogéneas. Esta discusión es importante, ya que al reconocer que la RILE fue construido a partir de una base deductiva, Budge (2013) se limitó a señalar las perspectivas teóricas de Marx, Engel y Lenin para justificar las categorías de izquierda. Sin embargo, como hemos demostrado, las categorías de izquierda que componen la RILE van más

Los estudios de Dinas y Gemenis (2009) y Budge (2013) comparan la efectividad del método estándar MARPOR con métodos alternativos, como los que cambian la base de conceptualización de la RILE (Jahn, 2010) que emplean una escala de proporción logit (Benoit et al, 2012b), y que aplican el análisis factorial a través de métodos inductivos (Gabel y Huber, 2000). Con respecto específicamente al método estándar, las conclusiones de esos estudios apuntan a diferentes rutas. Para Dinas y Gemenis (2009), el método estándar de MARPOR no es superior a los métodos alternativos. A su vez, Budge (2013) concluye que los investigadores deben atenerse al uso del método estándar si éste cumple sus objetivos de investigación. 
allá del marxismo ortodoxo, incorporando una miríada de perspectivas, entre las cuales podemos situar las keynesianas y kantianas.

\section{Análisis de los posicionamientos ideológicos}

En el contexto latinoamericano, la disputa ideológica central gira en torno a la adhesión -o no-, a las prescripciones neoliberales que involucran medidas vinculadas a "la adopción de la disciplina fiscal, la liberalización del comercio, la desregulación económica y la transferencia de servicios sociales al sector privado" (Oliveira, 2019: 12). Estas reformas se implementaron en dos oleadas diferentes: la primera iniciada por una reforma radical en Chile en la década de 1980 y la segunda, en la década de 1990, durante gobiernos democráticos que tenían como objetivo expandir los derechos políticos y sociales, lo que aumentó los ajustes de gasto fiscal y social (Draibe, 2002).

En Argentina, si las medidas iniciales adoptadas por el presidente Carlos Menem lograron controlar la inflación y promover el crecimiento económico a costa de privatizaciones y de la casi total desnacionalización de la economía, con un tipo de cambio atado al dólar y la entrada de recursos externos, al final de su mandato la deuda del país era equivalente el $50 \%$ del PIB (Bandeira, 2002). Incluso con las reformas radicales de Menem, el país fue encaminándose hacia una de sus más grandes depresiones. En los años siguientes, protestas masivas por el país, motivadas por los problemas económicos y sociales desencadenaron dos renuncias presidenciales sucesivas en pocos días, llevando la democracia del país al borde del abismo (Levitsky, 2005).

En Brasil el escenario económico y social no era muy diferente en el período después de la dictadura, con el empeoramiento de la crisis económica indicado por la caída industrial, el aumento de la tasa de desempleo en más del 6\%, la desvalorización del sueldo mínimo y el aumento de la concentración de la renta y la desigualdad social (Fleury, 1994). En la década del 90, el país había construido uno de los más grandes parques industriales y con Fernando Henrique Cardoso, presidente en dicha época, y el Plan Real, se contuvo la inflación, pero no la alta tasa de cambio que encareció los productos brasileños (Bandeira, 2002). Después de más de quince años desde la apertura democrática, Brasil aún presentaba problemas graves como la violencia urbana, el analfabetismo, una mala calidad de la educación, la oferta inadecuada de servicios de salud, desigualdades sociales y económicas (Carvalho, 2012). 
Chile, por otro lado, es tomado por gran parte de los investigadores como una de las democracias más exitosas de Latinoamérica (Hagopian, 2005). A diferencia de los otros países, no se enfrentaba a una crisis económica aguda que exigiera planes económicos de emergencia, aunque hubiera una expectativa por parte de la sociedad civil de algunas mejoras, tales como más empleo, acceso a la salud y educación, disminución de las desigualdades sociales e integración de los sectores marginalizados (Garretón, 1992).

Con la crisis mundial derivada de la burbuja inmobiliaria de los Estados Unidos, en el año 2008, países latinoamericanos sufrieron la baja de las exportaciones y del crédito externo, aumento de la tasa interés, retracción del mercado interno, baja de la producción y consecuente aumento del desempleo (Singer, 2009). Ante de esa coyuntura, enfrentaron la tarea difícil de promover el crecimiento económico y social. El resquebrajamiento de la hegemonía neoliberal y el retorno de la concepción del desarrollo económico abrieron espacio para la integración entre economía y políticas sociales, en la que la idea de Estado de bienestar es esencial (Draibe y Riesco, 2011). Así, al comprender al Estado de bienestar como un conjunto de derechos sociales garantizados por el Estado con el objetivo de promocionar cierta armonía entre desarrollo económico y estabilidad social (Gomes, 2006), no es raro que los partidos políticos, independientemente del espectro ideológico, hayan incorporado a lo largo de los años la garantía de tales derechos en sus discursos.

Programas Sociales como Bolsa Família en Brasil, encabezado por el Partido dos Trabalhadores (PT), tiene su continuidad defendida incluso por sus opositores. Protestas recientes en Chile contra el actual sistema de pensiones que excluiría a los trabajadores formales y contra la reforma de la educación superior; y las protestas de 2017 que terminaron en hechos violentos en Argentina contra la reforma de las jubilaciones propuesta por el gobierno de Macri, son importantes demostraciones de la demanda por parte de la sociedad de garantías mínimas contempladas por el Estado de bienestar.

Efectivamente, los partidos políticos de Brasil, Chile y Argentina han reproducido enfáticamente en sus manifiestos esas demandas, independientemente de sus posiciones ideológicas, de tal modo que, cuando quedan posicionados en la escala RILE del MARPOR, tienden a ocupar un espacio más a la izquierda de lo que generalmente son clasificados por los especialistas. A continuación, avanzamos con el análisis de esos casos.

Con respecto a Brasil, el MARPOR clasificó los manifiestos de los partidos que recibieron más votos en las elecciones ocurridas entre 1994 y 2014. Tal como demuestra el índice obtenido en la escala RILE, en la Tabla 2, a excepción del manifiesto del PSDB en la elección de 1994 (posicionado a la 
centro-derecha), todos los otros manifiestos codificados, en todas las elecciones analizadas de Brasil, fueron clasificados de la centro-izquierda a la izquierda. En contrapartida, el PSDB ha sido posicionado por investigadores como un partido de centro (Rodrigues, 2002). El PRONA, mientras existió, era clasificado como un partido de derecha (Mainwaring et al., 2000). Mientras tanto, PPS, PSB, PT son clasificados por los especialistas como centro-izquierda (Zucco, 2011), y el PSOL más a la izquierda (Zucco, 201 1; Tarouco y Madeira, 2013), lo que está en consonancia con las estimativas del MARPOR.

\section{TABLA 2}

Posición de los partidos brasileños en RILE y contribución (en\%) de las categorías de expansión del bienestar y expansión de la educación entre las categorías de izquierda.

\begin{tabular}{|c|c|c|c|c|c|c|}
\hline Partido/Coalición & Año & Candidato & RILE & per504 & per506 & Total Bienestar \\
\hline \multirow{7}{*}{ PT } & 1994 & Lula da Silva (PT) & -27.134 & 43.1 & 25.5 & 68.6 \\
\hline & 1998 & Lula da Silva (PT) & -19.905 & 29.4 & 17.6 & 47 \\
\hline & 2002 & Lula da Silva (PT) & -21.955 & 36.7 & 13.5 & 50.2 \\
\hline & 2006 & Lula da Silva (PT) & -19.005 & 35.1 & 23.9 & 59 \\
\hline & 2010 & Dilma Rousseff (PT) & -13.636 & 37.2 & 23.1 & 60.3 \\
\hline & 2014 & Dilma Rousseff (PT) & -12.048 & 30.3 & 32.4 & 62.7 \\
\hline & Promedio & & -18.947 & 35.3 & 22.7 & 58.0 \\
\hline \multirow{7}{*}{ PSDB } & 1994 & $\begin{array}{l}\text { Fernando Henrique Cardoso } \\
\text { (PSDB) }\end{array}$ & 4.843 & 29.8 & 30.6 & 60.4 \\
\hline & 1998 & $\begin{array}{l}\text { Fernando Henrique Cardoso } \\
\text { (PSDB) }\end{array}$ & -18.318 & 56.4 & 19.4 & 75.8 \\
\hline & 2002 & José Serra (PSDB) & -10.523 & 51.6 & 16.8 & 68.4 \\
\hline & 2006 & Geraldo Alckmin (PSDB) & -9.202 & 39.3 & 22.5 & 61.8 \\
\hline & 2010 & José Serra (PSDB) & -11.302 & 47.9 & 26.1 & 74 \\
\hline & 2014 & Aécio Neves (PSDB) & -5.085 & 44.0 & 31.5 & 75.5 \\
\hline & Promedio & & -8.265 & 44.8 & 24.5 & 69.3 \\
\hline \multirow{2}{*}{ PRONA } & 1994 & Enéas Carneiro (PRONA) & -27.333 & 43.0 & 37.0 & 80 \\
\hline & Promedio & & -27.333 & 43.0 & 37.0 & 80 \\
\hline \multirow{3}{*}{ PPS } & 1998 & Ciro Gomes (PPS) & -15.974 & 31.6 & 17.3 & 48.9 \\
\hline & 2002 & Ciro Gomes (PPS) & -20.711 & 21.0 & 16.5 & 37.5 \\
\hline & Promedio & & -18.342 & 26.3 & 16.9 & 43.2 \\
\hline \multirow{3}{*}{ PSB } & 2002 & Anthony Garotinho (PSB) & -12.53 & 19.8 & 38.5 & 58.3 \\
\hline & 2014 & Marina Silva (PSB) & -5.394 & 43.7 & 40.2 & 83.9 \\
\hline & Promedio & & -8.962 & 31.75 & 39.35 & 71.1 \\
\hline \multirow{2}{*}{ PSOL } & 2006 & Heloísa Helena (PSOL) & -51.128 & 10.4 & 4.2 & 14.6 \\
\hline & Promedio & & -51.128 & 10.4 & 4.2 & 14.6 \\
\hline \multirow{2}{*}{ PV } & 2010 & Marina Silva (PV) & -28.302 & 35.7 & 42.9 & 78.6 \\
\hline & Promedio & & -28.302 & 35.7 & 42.9 & 78.6 \\
\hline Todos & Promedio & & -17.086 & 36.1 & 25.2 & 61.3 \\
\hline
\end{tabular}

Fuente: Elaboración de los autores a partir de los datos de LEHMANN et al(2018). 
De todos modos, argumentamos que hasta los partidos de izquierda estarían posicionados más a la izquierda de su posición real. Por un lado, el desajuste entre las perspectivas teóricas que fundamentan las categorías de izquierda del MARPOR y su métrica imposibilitan la estimación de la posición de los partidos. Por el otro, las categorías asociadas al bienestar social actuarían como una fuerza centrífuga, empujando a los partidos más hacia la izquierda. Tal como muestran los datos de la Tabla 2, entre las elecciones de 1994 y 2014, las categorías "Expansión del Estado de bienestar" y "Expansión de la Educación" representaron un promedio de $61.3 \%$ entre todas las posiciones de izquierda del PT, PSDB, PRONA, PSB, PPS, PSOL y PV. Específicamente, la categoría "Expansión del Estado de bienestar" fue la más frecuente entre las categorías de izquierda en los manifiestos del PT en 1994, 1998, 2006 y 2010; del PSDB en 1998, 2002, 2006, 2010 y 2014; del PRONA en 1994; del PPS en 1998 y 2002, del PV en 2010 y del PSB en 2014. La categoría "Expansión de la Educación" fue la categoría de izquierda más frecuente en los manifiestos del PSDB en 1994, del PSB en 2002 y del PT en 2014.

Además, la comparación del impacto de las categorías de bienestar social sobre las posiciones del PT y del PSDB refuerza nuestro argumento. En los manifiestos del PSDB las categorías "Expansión del Estado de bienestar" y "Expansión de la Educación" representaron un promedio del 69.3\% de sus posiciones de izquierda, mientras que en el PT las mismas categorías representaron alrededor del $58 \%$ de sus posiciones de izquierda. De este modo, a pesar de que el PSDB ha sido clasificado por investigadores siempre a la derecha del PT (Rodrigues, 2002; Mainwaring, et al., 2000; Zucco, 2011), es evidente que las categorías relacionadas con el bienestar social posicionan al PSDB más hacia la izquierda de su ubicación real. De hecho, las estimaciones del MARPOR confirman, en parte, los análisis de los investigadores, resultando PSDB posicionado a la derecha del PT. Sin embargo, se destaca que en las estimaciones del MARPOR tanto el PSDB (-8.265) como el PT (-18.947) aparecen en la centro-izquierda.

En el caso chileno, el MARPOR codificó los manifiestos de las principales coaliciones que disputaron las elecciones presidenciales del país entre 1989 y 2013. Solamente los manifiestos de miembros de la coalición de la Alianza en las elecciones de 1989, 1993 y 2005 fueron posicionados en la centro-derecha en la RILE. Los otros manifiestos, de las otras coaliciones en todas las elecciones, fueron posicionados en la centro-izquierda. En el promedio del período, solo la Alianza está posicionada en la centro-derecha. Especialistas, por su parte, han clasificado a la Concertación (desde 2013 "Nueva Mayoría”) en la centro-izquierda; la Alianza y sus miembros indivi- 
dualmente, Unión Demócrata Independiente (UDI) y Renovación Nacional (RN), a la derecha (Rami y Leal, 2014); Juntos Podemos Más, (Rami y Leal, 2014); y Si Tú quieres, Chile cambia, a la izquierda (Dosek y Freindeberg, 2014) y la candidatura independiente de Francisco Javier Errázuriz en 1989, al centro (Klugmann, 1991).

\section{TABLA 3}

Posición de los partidos chilenos en la RILE y contribución (en \%) de las categorías de expansión del bienestar y expansión de la educación entre las categorías de izquierda

\begin{tabular}{|c|c|c|c|c|c|c|}
\hline Partido/Coalición & Año & Candidato & RILE & per504 & per506 & Total Bienestar \\
\hline \multirow{7}{*}{$\begin{array}{l}\text { Concertación/ } \\
\text { Nueva Mayoría }\end{array}$} & 1989 & Patricio Aylwin (PDC) & -15.152 & 12.7 & 19.9 & 32.6 \\
\hline & 1993 & Eduardo Frei Ruiz -Tagle (PDC) & -10.493 & 20.2 & 31.8 & 52 \\
\hline & 1999 & Ricardo Lagos Escobar (PPD) & -13.596 & 37.9 & 29.7 & 67.6 \\
\hline & 2005 & Michelle Bachelet Jeria (PS) & -19.715 & 36.5 & 15.8 & 52.3 \\
\hline & 2009 & Eduardo Frei Ruiz-Tagle (PDC) & -18.857 & 38.9 & 19.4 & 58.3 \\
\hline & 2013 & Michelle Bachelet Jeria (PS) & -18.974 & 32.5 & 26.1 & 58.6 \\
\hline & Promedio & & -16.131 & 29.8 & 23.8 & 53.6 \\
\hline \multirow{8}{*}{ Alianza } & 1989 & Hernán Büchi (ind) & 21.405 & 23.0 & 27.8 & 50.8 \\
\hline & 1993 & Arturo Alessandri Besa (UDI) & 25.347 & 25.7 & 31.9 & 57.6 \\
\hline & 1999 & Joaquín Lavín Infante (UDI) & -5.017 & 38.3 & 29.2 & 67.5 \\
\hline & 2005 & Joaquín Lavín Infante (UDI) & 16.892 & 28.0 & 56.0 & 84 \\
\hline & 2005 & Sebastián Piñera Echenique (RN) & -0.809 & 28.6 & 31.0 & 59.6 \\
\hline & 2009 & Sebastián Piñera Echenique (RN) & -3.029 & 29.6 & 29.6 & 59.2 \\
\hline & 2013 & Evelyn Matthei (UDI) & -28.011 & 32.6 & 35.7 & 68.3 \\
\hline & Promedio & & 4.598 & 29.4 & 34.5 & 63.9 \\
\hline \multirow{2}{*}{ Independiente } & 1989 & Francisco Javier Errázuriz (ind) & -3.968 & 58.3 & 4.2 & 62.5 \\
\hline & Promedio & & -3.968 & 58.3 & 4.2 & 62.5 \\
\hline \multirow{3}{*}{$\begin{array}{c}\text { Juntos Podemos } \\
\text { Más }\end{array}$} & 2005 & Tomás Hirsch Goldschmidt (PH) & -36.323 & 22.3 & 14.5 & 36.8 \\
\hline & 2009 & Jorge Arrate (PCCh) & -41.486 & 25.3 & 13.8 & 39.1 \\
\hline & Promedio & & -38.9 & 23.8 & 14.2 & 38.0 \\
\hline \multirow{2}{*}{$\begin{array}{c}\text { Nueva Mayoría } \\
\text { para Chile }\end{array}$} & 2009 & Marco Enríquez-Ominami (ind) & -28.325 & 37.4 & 18.4 & 55.8 \\
\hline & Promedio & & -28.325 & 37.4 & 18.4 & 55.8 \\
\hline \multirow{2}{*}{$\begin{array}{l}\text { Si tú quieres, } \\
\text { Chile cambia }\end{array}$} & 2013 & Marco Enríquez-Ominami (PRO) & -37.011 & 21.7 & 19.8 & 41.5 \\
\hline & Promedio & & -37.011 & 21.7 & 19.8 & 41.5 \\
\hline Todos & Promedio & & -12.062 & 30.5 & 25.3 & 55.8 \\
\hline
\end{tabular}

Fuente: Elaboración de los autores a partir de los datos de Lehmann et al (2018).

Igual que en el caso brasileño, las categorías "Expansión del Estado de bienestar" y "Expansión de la Educación" representaron la mayoría (un promedio de $55.8 \%$ ) de las posiciones de izquierda de las coaliciones Concertación/Nueva Mayoría, de la Alianza, de la candidatura de Francisco 
Javier Errázuriz en 1989, de Juntos Podemos Más, de Nueva Mayoría para Chile y de Si tú quieres, Chile cambia. Particularmente, la categoría "Expansión del Estado de bienestar" fue la más frecuente entre las categorías de izquierda en los manifiestos de la Concertación en 1999, 2005, 2009, así como de Nueva Mayoría, en 2013; de laAlianza en 1999 y 2009; de la candidatura independiente de centro en 1989; de Nueva Mayoría para Chile y de Juntos Podemos Más en 2009. Por su parte, la categoría "Expansión de la Educación" fue la posición de izquierda más frecuente en los manifiestos de la Concertación en 1993; de la Alianza en 1993, 2009, 2013 y de sus miembros Unión Demócrata Independiente (UDI) y Renovación Nacional (RN), en 2005 cuando disputaron las elecciones separadamente.

También comparamos la contribución de las categorías de bienestar social en las posiciones de izquierda de las principales coaliciones. Aunque, generalmente, la posición de la Alianza en la RILE está de conformidad con la clasificación de especialistas, las categorías referentes al bienestar social la empujan a la izquierda de su posición real. Efectivamente, en los manifiestos de la Alianza, las categorías "Expansión del Estado de bienestar" y "Expansión de Educación" representaron un promedio del $63.9 \%$ de sus posiciones de izquierda, mientras que en la Concertación/Nueva Mayoría las mismas categorías representaron un promedio del $53.6 \%$ de sus posiciones de izquierda.

En relación con Argentina, el MARPOR codificó las elecciones presidenciales que ocurrieron entre 1995 y 2011. Allí, a diferencia de los casos brasileño y chileno, las coaliciones partidarias no deben ser el único criterio para evaluar comparativamente las mediciones de la escala RILE. De este modo, dividimos los manifiestos argentinos considerando también la recurrencia de candidaturas, reconociendo el carácter importante del personalismo en el sistema político del país. Entre los veinte manifiestos codificados por el MARPOR, apenas cuatro fueron posicionados en la centroderecha (aunque muy próximos al centro absoluto). Esto son: el manifiesto de Carlos Menem del Partido Justicialista (PJ) de 1995; de Adolfo Rodríguez Sáa, en 2003, de Alberto Rodríguez Saá en 2011 (dos hermanos que constituyeron un peronismo disidente, identificado como peronismo federal); y de Domingo Cavallo del Partido Acción por la República, en 1999. Los demás manifiestos fueron posicionados dela centro-izquierda a la izquierda por el MARPOR. 
Flávio Contrera, Priscilla Leine Cassotta y Matheus Lucas Hebling

\section{TABLA 4}

Posición de los partidos argentinos en la RILE y contribución (en \%) de las categorías de expansión del bienestar y expansión de la educación entre las categorías de izquierda

\begin{tabular}{|c|c|c|c|c|c|c|}
\hline Partido/Coalición & Año & Candidato & RILE & per504 & per506 & $\begin{array}{c}\text { Total } \\
\text { Bienestar }\end{array}$ \\
\hline \multirow{12}{*}{$\mathrm{PJ}$} & 1995 & Carlos Menem (PJ) & 1.997 & 22.8 & 20.7 & 43.5 \\
\hline & 2003 & Carlos Menem (PJ) & -7.958 & 27.6 & 28.9 & 56.5 \\
\hline & Promedio & & -2.981 & 25.2 & 24.8 & 50 \\
\hline & 1999 & Eduardo Duhalde (PJ) & -6.644 & 26.2 & 24.1 & 50.3 \\
\hline & 2011 & Eduardo Duhalde (PJ) & -50 & 14.6 & 14.6 & 29.2 \\
\hline & Promedio & & -3.347 & 20.4 & 19.35 & 39.75 \\
\hline & 2003 & Néstor Kirchner (PJ) & -22.667 & 15.0 & 20.0 & 35 \\
\hline & 2007 & Christina Kirchner (PJ) & -20.755 & 16.7 & 22.2 & 38.9 \\
\hline & 2011 & Christina Kirchner (PJ) & -3.425 & 14.6 & 14.6 & 29.2 \\
\hline & Promedio & & -15.616 & 15.4 & 18.9 & 34.4 \\
\hline & 2003 & $\begin{array}{l}\text { Adolfo Rodríguez Saá } \\
\text { (PJ) }\end{array}$ & 4.167 & 50.0 & 0.0 & 50 \\
\hline & 2007 & $\begin{array}{c}\text { Alberto Rodríguez Saá } \\
\text { (PJ) }\end{array}$ & -26.923 & 40.0 & 40.0 & 80 \\
\hline \multirow[t]{2}{*}{ Es Posible } & 2011 & $\begin{array}{l}\text { Alberto Rodríguez Saá } \\
\text { (EP) }\end{array}$ & 1.245 & 38.0 & 27.8 & 65.8 \\
\hline & Promedio & & -7.170 & 42.7 & 22.6 & 65.3 \\
\hline \multirow{3}{*}{ UCR } & 1995 & $\begin{array}{c}\text { Horacio Massaccesi } \\
\text { (UCR) }\end{array}$ & -11.136 & 12.5 & 19.8 & 32.3 \\
\hline & 2011 & Ricardo Afonsín (UCR) & -15.094 & 32.1 & 35.7 & 67.8 \\
\hline & Promedio & & -13.115 & 22.3 & 27.75 & 50.05 \\
\hline \multirow{2}{*}{ FREPASO } & 1995 & José Octavio Bordón & -15.217 & 28.6 & 21.4 & 50 \\
\hline & Promedio & $($ PAIS $)$ & -15.217 & 28.6 & 21.4 & 50 \\
\hline \multirow{2}{*}{ ALIANZA } & 1999 & Fernando de la Rúa & -13.04 & 29.9 & 17.4 & 47.3 \\
\hline & Promedio & (UCR) & -13.04 & 29.9 & 17.4 & 47.3 \\
\hline \multirow{2}{*}{ UNA } & 2007 & \multirow{2}{*}{ Roberto Lavagna (ind) } & -13.213 & 38.5 & 11.5 & 50 \\
\hline & Promedio & & -13.213 & 38.5 & 11.5 & 50 \\
\hline \multirow{2}{*}{$\begin{array}{l}\text { Acción por la } \\
\text { República }\end{array}$} & 1999 & Domingo Cavallo & 0.176 & 37.2 & 21.7 & 58.9 \\
\hline & Promedio & (Acc. por la República) & 0.176 & 37.2 & 21.7 & 58.9 \\
\hline \multirow{2}{*}{ RECREAR } & 2003 & Ricardo López Murphy & -0.381 & 8.4 & 77.0 & 85.4 \\
\hline & Promedio & (RECREAR) & -0.381 & 8.4 & 77.0 & 85.4 \\
\hline \multirow{3}{*}{$\begin{array}{c}\text { Coalición Cívica - } \\
\text { ARI }\end{array}$} & 2003 & Elisa Carrió (ARI) & -26.135 & 33.8 & 15.6 & 49.4 \\
\hline & 2007 & Elisa Carrió (ind) & -11.006 & 28.9 & 14.1 & 43 \\
\hline & Promedio & & -18.571 & 31.35 & 14.85 & 46.2 \\
\hline \multirow{2}{*}{$\begin{array}{l}\text { Frente Amplio } \\
\text { Progresista }\end{array}$} & 2011 & \multirow{2}{*}{ Hermes Binner (PS) } & -17.351 & 39.0 & 24.1 & 63.1 \\
\hline & Promedio & & -17.351 & 39.0 & 24.1 & 63.1 \\
\hline Todos & Promedio & & -12.668 & 27.7 & 23.6 & 51.5 \\
\hline
\end{tabular}

Fuente: Elaboración de los autores a partir de los datos de Lehmann et al (2018). 
En ese momento es importante comparar las estimaciones de posición del MARPOR con estimaciones de especialistas. De hecho, éstos han clasificado al menemismo en la derecha (Arditi, 2009), y al peronismo federal de los hermanos Rodríguez Saá (Retamozo y Schuttenberg 2016) y al partido de Domingo Cavallo en la centro-derecha (Camou, 2000). Aunque estas clasificaciones estén en consonancia con las estimaciones del MARPOR, hay que considerar el hecho de que el MARPOR ha posicionado a esos frentes y partidos muy próximos al centro absoluto. Por su parte, el Partido Recrear, clasificado por especialistas en la centro-derecha (Moreira, 2006), fue posicionado por el MARPOR en la centro-izquierda. Además, frentes que apoyaron a Eduardo Duhalde, generalmente clasificados por especialistas en el centro (Retamozo y Schuttenberg, 2016), fueron posicionados en la centro-izquierda por el MARPOR. Asimismo, el Frente Amplio Progresista de Binner, clasificado por especialistas como centro "gorila"-antiperonista(Retamozo y Schuttenberg, 2016) fue ubicado por el MARPOR en la centro-izquierda. Por otra parte, el MARPOR confirmó las clasificaciones de especialistas acerca de la posición en la centro-izquierda del Frente para la Victoria de los Kirchner (Saéz, 2008; Arditi, 2009), de las candidaturas de la UCR, del FREPASO, de de la Alianza, y del Partido Coalición Civica ARI de Elisa Carrió (Moreira, 2006).

Entre las elecciones de 1995 y 2011, las categorías "Expansión del Estado de bienestar" y "Expansión de la Educación" representaron un promedio del $51.5 \%$ de todas las posiciones de izquierda de los frentes que apoyaron, en el universo del PJ, a las candidaturas de Menem, Duhalde, de los Kirchner, y de los hermanos Rodríguez Saá; así como también a las candidaturas de la UCR, del FREPASO, de la Alianza, de Una Nación Avanzada, de Acción por la República, de Recrear, de la Coalición Cívica ARI y del Frente Amplio Progresista. Específicamente, la categoría "Expansión del Estado de bienestar" fue la más frecuente entre las categorías de izquierda en los manifiestos de Menem y del FREPASO en 1995; de los frentes de apoyo a Duhalde en 1999 y 2011; de la Alianza en 1999, de Una Nación Avanzada en 2007 y de la Unión Cívica Radical en 2011; de Acción por la República en 1999; de los frentes disidentes de los hermanos Rodríguez Saá en 2003, 2007 y 2011 ; de ARI (Coalición Cívica) en 2003 y 2007; y del Frente Amplio Progresista de 2011. A su vez, la categoría "Expansión de la Educación" fue la más frecuente entre las categorías de izquierda en los manifiestos del Frente de Menem y del Partido Recrear en 2003, y del Frente para la Victoria de Kirchner y de la FREJULI de Alberto Rodríguez Saá en 2007.

$\mathrm{Al}$ igual que en los casos de Brasil y Chile, las categorías referidas al bienestar social fueron las categorías de izquierda más enfatizadas por los 
partidos, frentes ycoaliciones argentinas de centro-derecha. De este modo, es posible destacar el impacto de las categorías "Expansión del Estado de bienestar" y "Expansión de la Educación" en los manifiestos de Recrear, de los frentes liderados por los hermanos Rodríguez Saá, de Acción por la República y de los frentes de apoyo a Menem. Aunque todos esos partidos, frentes y coaliciones fueron clasificados por especialistas a la derecha del Frente por la Victoria de los Kirchner, el impacto de las categorías de bienestar social del MARPOR fue más grande en esos frentes de que en los manifiestos de los Kirchner.

\section{TABLA 5}

Posición media de manifiestos en RILE por país y contribución (en\%) de las categorías de expansión del bienestar y expansión de la educación entre las categorías de izquierda en las últimas cinco elecciones para el Ejecutivo

\begin{tabular}{lcccc}
\hline País & RILE & per504 & per506 & $\begin{array}{c}\text { Total } \\
\text { Bienestar }\end{array}$ \\
\hline Suecia & -6.669 & 37.9 & 26.1 & 64.0 \\
Brasil & -17.086 & 36.1 & 25.2 & 61.3 \\
Dinamarca & -1.861 & 35.4 & 23.8 & 59.2 \\
Finlandia & -8.835 & 40.2 & 17.0 & 57.1 \\
Noruega & -11.301 & 32.2 & 23.6 & 55.8 \\
Chile & -12.062 & 30.5 & 25.3 & 55.8 \\
Luxemburgo & -14.131 & 32.4 & 23.1 & 55.5 \\
Reino Unido & -1.308 & 33.2 & 19.9 & 53.1 \\
Irlanda & -14.605 & 37.0 & 15.7 & 52.7 \\
Argentina & -12.668 & 27.7 & 23.6 & 51.5 \\
Italia & 5.524 & 31.0 & 19.3 & 50.3 \\
Holanda & 5.947 & 25.4 & 24.8 & 50.2 \\
Islandia & -7.981 & 31.9 & 17.3 & 49.2 \\
Bélgica & -5.869 & 37.6 & 10.1 & 47.7 \\
Francia & -11.094 & 24.7 & 17.5 & 42.3 \\
Estados & 15.5 & 21.1 & 17.9 & 39.0 \\
Unidos & & & 17.3 & 38.9 \\
Austria & -10.385 & 21.6 & 14.9 & 30.7 \\
Alemania & -6.792 & 15.7 & & \\
\hline
\end{tabular}

Fuente: Elaboración de los autores a partir de los datos de Lehmann et al (2018). 
A partir de lo expuesto, no cabe duda de que las categorías "Expansión del Estado de bienestar" y "Expansión de la Educación" contribuyen fuertemente al posicionamiento ideológico más a la izquierda de partidos argentinos, brasileños y chilenos en la RILE. Considerando que la RILE fue construida con base en el contexto político europeo y que este es fundamentalmente distinto del contexto sudamericano, $\operatorname{comparamos}^{6}$, las medias de posición en la RILE y de la contribución de las categorías "Expansión del Estado de bienestar" y "Expansión de la Educación", entre aquellos tres países sudamericanos y catorce naciones de Europa Occidental. Incluimos también a los Estados Unidos, conformando un grupo de 15 democracias industriales avanzadas, tal como muestra la Tabla 5.

Respecto a la RILE, Brasil es el país posicionado más a la izquierda entre los 18 países analizados. Argentina es el cuarto y Chile, por su parte, es el quinto país posicionado más a hacia la izquierda; los manifiestos posicionados más a hacia la derecha son de Estados Unidos, Holanda e Italia.

Con relación al peso de las categorías "Expansión del Estado de bienestar" y "Expansión de la Educación" entre las categorías de izquierda de la RILE, una vez más destacamos los manifiestos de los partidos sudamericanos. En los manifiestos de Brasil (solamente detrás de Suecia) y Chile (en el quinto lugar), la contribución de esas dos categorías es equivalente a la de los países escandinavos, los cuales tradicionalmente están asociados a las cuestiones de bienestar social. Más abajo aparece la Argentina, pordelante de ocho países del grupo analizado. Efectivamente, es evidente en esa comparación el peso que las categorías de bienestar social presentan en los manifiestos sudamericanos.

\section{Conclusiones}

A partir del análisis propuesto, nuestra contribución fue verificar que el método estándar del MARPOR para estimar la posición ideológica de los partidos políticos en espacios de competencia presenta una serie de imprecisiones relacionadas con las categorías que componen la escala RILE. Dos de esas imprecisiones son de orden metodológico y afectan la estimación de la posición ideológica de todos los partidos. La primera imprecisión es la de que no todas las categorías que forman la escala RILE son comparables. Luego, se comparan énfasis en posiciones de los partidos sobre asun-

\footnotetext{
$6 \quad$ Para ello seleccionamos todos los manifiestos codificados por el MARPOR en las últimas cinco elecciones para el Ejecutivo (sean presidenciales o parlamentarias) en los quince países incluídos en el análisis.
} 
tos diferentes. La segunda imprecisión se refiere a la manera del conteo de las categorías, en tanto no refleja las variaciones ideológicas presentes en las perspectivas teóricas; es decir, categorías como "Antiimperialismo" o "Expansión del Estado de bienestar" son contabilizadas de la misma manera, aunque la primera esté, desde el punto de vista teórico e ideológico, posicionada más a la izquierda que la segunda.

Hay otra imprecisión, relacionada con la aplicación del método estándar de estimación para partidos de Brasil, Chile y Argentina. El análisis de esta estimación reveló que independientemente de la clasificación que los partidos de esos países reciben de los especialistas, éstos han sido posicionados más hacia la izquierda por los investigadores del MARPOR. Respecto de esto, verificamos que los partidos de esos países tienden, entre las categorías de izquierda, a enfatizar en los asuntos "Expansión del Estado de bienestar" y "Expansión de la Educación", los cuales empujan a los partidos sudamericanos más hacia la izquierda.

La extensión de la aplicación del método del MARPOR para los países sudamericanos y la validez de las categorías de izquierda representan desafíos para la adaptación del método fuera del contexto europeo. Agrupar diferentes países como Argentina, Brasil y Chile en un mismo análisis no es una tarea fácil de lograr. América Latina ha sido colonizada por diferentes países y cada pueblo nativo tenía sus propias características. Además, obedece a diferentes patrones de modernización, que van desde las características modernas establecidas inicialmente con la inmigración tardía como en Argentina, Uruguay y el estado de San Pablo en Brasil hasta las colonias pobres en el margen de los imperios, como es el caso de Chile (Draibe y Riesco, 2007). Por esta razón, es posible que la adaptación nunca traduzca completamente las especificidades de cada uno de estos países. Se trata de una desventaja del comparativismo que intentaremos superar a través de elecciones basadas en la teoría, que harán que las categorías MARPOR sean más sólidas.

Al señalar las limitaciones del método de MARPOR, nuestros hallazgos dialogan con los resultados de otros trabajos críticos a la precisión de sus estimaciones ideológicas. Parte de la literatura critica la tendencia a la centralización de las posiciones partidarias del método (Lowe et al, 2011; Benoit et al, 2012a), mientras otros objetivan la gran variabilidad de las clasificaciones de partidos más extremos (Slapin y Proksch, 2008). Budge y Meyer (2014) realizaron esfuerzos para intentar resolver parte de ese problema, fijándose principalmente en la capacidad de tales categorías para medir a largo plazo las preferencias políticas de los partidos. Por otro lado, críticas al método apuntan a la incapacidad de los manifiestos para representar posiciones reales (Hansen, 2008; Dinas y Gemenis, 2009; Gemenis, 2013). 
A causa de esas imprecisiones, concluimos que el método estándar del MARPOR debe ser utilizado con cautela para interpretar las posiciones ideológicas de los manifiestos de los países analizados. No se trata de descartar las estimaciones realizadas por el MARPOR, sino, como sugirieron Dinas y Gemenis (2009), de interpretarlas junto con otras fuentes de datos, tales como clasificaciones de expertos, encuestas de opinión pública sobre los partidos y análisis de votaciones nominales en el legislativo; o como lo sugieren López et al (2013), interpretar el eje izquierda-derecha en conjunto con otros ejes socio-económicos.

Siguiendo una orientación metodológica deductiva, vinculamos las categorías de izquierda del MARPOR con discusiones teóricas de corrientes plurales. Antes de proponer cualquier medida alternativa, entendemos que ese mismo emprendimiento teórico debe realizarse en relación con las categorías de derecha. En este sentido, nuestro trabajo se limita a señalar las imprecisiones de estimación ideológica del MARPOR y a entender las categorías de análisis de izquierda a partir de una orientación deductiva. Creemos que cualquier intento futuro de reajuste de la RILE debe primordialmente considerar la construcción de una escala compuesta exclusivamente de categorías comparables, cuya medición reciba pesos distintos de conformidad con las variaciones ideológicas expresadas en las perspectivas teóricas. Con respecto a la aplicación del método para la estimación de la posición de partidos en América del Sur, sugerimos además que las categorías incluidas en la RILE reflejen con mayor precisión los conflictos de la disputa izquierda-derecha en el contexto local.

\section{Referencias bibliográficas}

Aldrich, J. H. (2011). Why Parties? A Second Look. Chicago y Londres: University of Chicago Press.

Arditi, B. (2009). El giro a la izquierda en América Latina: cuna política post-liberal? Ciências Sociais Unisinos, 45(3), 232-246.

Ares, C. y A. Volkens (2017). ¿Por qué y cómo se está extendiendo el Manifesto Project a América Latina? Revista Española de Ciencia Política, 43,115-135.

Bandeira, L. M. (2002). As políticas neoliberais e a crise na América do Sul. Revista Brasileira de Política Internacional 45(2), 135-146.

Benoit, K., M. Laver, W. Lowe, y S. Mikhaylov (2012a). How to scale coded text units without bias: A response to Gemenis. Electoral Studies 31(3), 605-8. 
Botz, D. L. (2013). The Marxist View of the Labor Unions: Complex and Critical. The Journal of Labor and Society 16(1), 5-41.

Budge, I. (1982). Electoral volatility: Issue effects and basic change in 23 post-war democracies. Electoral Studies 1(2), 147-168.

Budge, I. (1987). The Internal Analysis of Election Programmes. En I. Budge, D. Robertson y D J. Hearl (Eds.), Ideology, Strategy and Party Change. Cambridge: Cambridge University Press, 15-38.

Budge, I. (2013). The standard Right-Left scale. Manifesto Project Paper. Disponible en: $<$ https://manifestoproject.wzb.eu/down/papers/budge_right-left-scale.pdfight-leftscale.pdf $>$

Budge, I. y T, Meyer (2014). «Understanding and Validating the Left-Right Scale (RILE)». En Volkens, A. et al., Mapping Policy Preferences From Texts: Statistical Solutions for Manifesto Analysts. Oxford: Oxford University Press.

Camou, A. (2000). ¿Del bipartidismo al bialiancismo? Elecciones y política en la Argentina posmenemista. Perfiles Latinoamericanos, 8(16), 11-30.

Carvalho, J. M. (2012). Cidadania no Brasil: O longo caminho. Río de Janeiro: Civilização Brasileira.

D'Alessandro, M. (2013). Las plataformas electorales en la Argentina moderna. América Latina Hoy, 65, 107-139.

Dinas, E. y K. Gemenis. (2009). Measuring Parties' Ideological Positions with Manifesto Data: A Critical Evaluation of the Competing Methods. Party Politics, 16(4), 427450.

Dosek, T. y F. Friendeberg. (2014). Voto hacia nuevos candidatos en sistemas de partidos (des)institucionalizados: el caso de Marco Enríquez-Ominami en Chile 2009. En Revista SAAP 8(1), 11-42.

Downs, A. (1957). An Economic Theory of Democracy. Nueva York: Harper.

Draibe, S. M. (2002). Brasil, 1980-2000. Proteção e insegurança sociais em tempos difíceis. Cadernos NEPP, 65, 1-64.

Draibe, S. M. y M. Riesco. (2011). Estados de Bem-Estar Social e estratégias de desenvolvimento na América Latina. Um novo desenvolvimentismo em gestação? Sociologias, 13(27), 220-254.

Draibe, S. M. y M. Riesco. (2007) Introducción y Capítulo 1. En: Riesco, M. (Ed.), Latin America: a new developmental welfare state model in the making? Londres: Palgrave MacMillan.

Fleury, S. (1994). Estado sem cidadãos: seguridade social na América Latina. Río de Janeiro: Editora Fiocruz.

Gabel, M. J. y J. D. Huber. (2000). Putting parties in their place: Inferring party leftright ideological positions from party manifestos data. American Journal of Political Science, 44(1), 94-103. 
Garretón, M. A. M. (1992). A Redemocratização no Chile: Transição, inauguração e evolução. Revista Lua Nova, 27, 59-92.

Gemenis, K. (2013). What to Do (and Not to Do) with the Comparative Manifestos Project Data. Political Studies, 61(S1), 3-23.

Gomes, F. G. (2006). Conflito social e welfare state: Estado e desenvolvimento social no Brasil. RAP, 40(2), 201-36.

Hagopian, F. (2005). “Government Performance, Political Representation, and Public Perceptions of Contemporary Democracy in Latin America”. En F. Hagopian y S. Mainwaring (Eds.), The Third Wave of Democratization in Latin America: Advances and Setbacks. Cambridge: Cambridge University Press.

Hansen, M. (2008). Back to the Archives? A Critique of the Danish Part of the Manifesto Dataset. Scandinavian Political Studies, 31(2), 201-216.

Hastings, A., A. Mason y H. Pyper. (2000). The Oxford Companion to Christian Thought. Oxford: Oxford University Press.

Jahn, D. (2010). Conceptualizing Left and Right in comparative politics: Towards a deductive approach. Political Parties, 17(6), 1-21.

Kant, I. (2003). To perpetual peace: a philosophical sketch. Hackett Publishing.

Keynes, J. M. (1936). The General Theory of Employment, Interest and Money. Londres: Palgrave Macmillan.

Klingemann, H. D., A. Volkens, J. Bara, J., I. Budge, I. y M. Mcdonald. (2006). Mapping Policy Preferences II. Estimates for Parties, Electors, and Governments in Eastern Europe, the European Union and the OECD, 1990-2003. Oxford: Oxford University Press.

Klugmann, M. (1991). La paradoja de la mayoria electoral: ¿donde esta el centro? Estudios Publicos, 42, 135-153.

Laver, M. y I. Budge. (1992). Measuring Policy Distances and Modelling Coalition Formation. En M.

Laver y I. Budge (Eds.) (1992). Party Policy and Government Coalitions. Basingstoke: Macmillan, 15-40.

Lenin, V. (2010). Imperialism, the Highest Stage of Capitalism. Londres: Penguin Classics.

Lehmann, P., T. Matthieß, N. Merz, S. Regel y A. Werner. (2018). Manifesto Corpus Database. Version: 2017-2. Berlin: WZB Berlin Social Science Center.

Levitsky, S. (2005). Argentina: Democratic Survival amidst Economic Failure. En F. Hagopian y S. Mainwaring (Eds.), The Third Wave of Democratization in Latin America: Advances and Setbacks. Cambridge: Cambridge University Press.

López, M. Á., N. Miranda y P. V. Gutiérrez. (2013). Estimando el espacio político del Cono Sur y Brasil: las elecciones presidenciales en el eje izquierda-derecha. POSTData: Revista de Reflexión y Análisis Político, 18(2), 403-442.

Lowe, W., K. Benoit, S. Mikhaylov, y M. Laver (2011). Scaling Policy Preferences from Coded Political Texts. Legislative Studies Quarterly, 36(1),123-55. 
Luxemburg, R. (1954). What is Economics? Nueva York: Pioneer Publishers.

Mainwaring, S., T. Power y R. Meneguello (2000). Partidos conservadores no Brasil contemporâneo: quais são, o que defendem, quais são suas bases. São Paulo: Paz e Terra.

Manoilescu, M. (1929). Théorie de protectionnisme et de l'échange international. París: Marcel Girard.

Marx, K. y F. Engels (1963). The Communist Manifesto. Nueva York: Russell \& Russell, Inc.

Mella, M., P. Valenzuela, M. López-Varas y C. Berrios (2019). Cambios programáticos y estratégicos de la derecha en Chile (1989-2013). Colombia Internacional, 100,91-120.

Moreira, C. (2006). Sistemas de partidos, alternancia política e ideología en el cono sur. Revista Uruguaya de Ciencia Política, 15, 31-56.

Nenovsky, N. N. y D. Torre (2015). Productivity-Based Protectionism: A Marxian Reconstruction of Mihail Manoilescu's Theory.Journal of Economic Issues, 49(3), 772-786.

Oliveira, A. N. C. (2019). Organização e ideologia nos partidos da américa latina: uma aproximação da hipótese de Michels. DADOS, 62(1), 1-38.

Petrocik, J. (1996). Issue ownership in presidential elections, with a 1980 case study. American Journal of Political Science, 40(3), 825-850.

Posner, R. A. (1974). Theories of Economic Regulation. Bell Journal of Economics and Management Science, 5(2), 335-358.

Ramil, M. A. F. y F. R. Leal (2014). Las elecciones presidenciales y parlamentarias chilenas 2009-2010: entre la sorpresa electoral y los desafíos políticos. POSTData, 19(1),11-44.

Retamozo, M. y M. Schuttenberg. (2016). La política, los partidos y Las elecciones en Argentina 2015: chacia un cambio en el campo político? Análisis politico, 86, 113-140.

Robertson, D. (1976). A Theory of Party Competition. Londres: Wiley.

Rodrigues, L. M. (2002). Partidos, Ideologia e Composição Social. RBCS, 17(48), $31-47$.

Sáez, A. M. (2008). La escala de la izquierda. La ubicación ideológica de presidentes y partidos de izquierda en América Latina. En Nueva Sociedad 212, 73-85.

Singer, P. (2009). A América Latina na Crise Mundial. Revista Estudos Avançados, 23(66), 91-102.

Slapin, J. B., y Proksch, S.-O. (2008). A Scaling Model for Estimating Time-Series Party Positions from Texts. American Journal of Political Science, 52(3):705-22.

Stigler, G. J. (1971). The Theory of Economic Regulation. En Bell Journal of Economics and Management Science, 2(1), 3-21.

Tarouco, G. (2011). Brazilian parties according to their manifestos: political identity and programmatic emphases. Brazilian Political Science Review, 5(1), 54-76. 
Tarouco, G. y R. Madeira (2013). Partidos, programas e o debate sobre esquerda e direita no Brasil. Revista Sociologia e Polítíca, 21(45), 149-165.

Tarouco, G. y R. Madeira. (2015). Os partidos brasileiros segundo seus estudiosos: Análise de um expert survey. Civitas - Revista de Ciências Sociais, 15(1),24-39.

Tavits, M. (2007). Principle vs. pragmatism: policy shifts and political competition. American Journal of Political Science, 51(1), 151-165.

Volkens, A., C. Ares, C., R. Bratanova y L. Kaftan (2015). Análisis de contenido de textos con referencias al Manifesto Project (2000-2015): temas, actores, y métodos para medir preferencias políticas. RIPS, 14(2), 233-238.

Werner, A., O. Lacewell y A. Volkens (2015). Manifesto Coding Instructions: 5th fully revised edition.

Manifesto Project Paper. Disponible en: <https://manifesto-project.wzb.eu/down/ papers/handbook_2014_version_5.pdf >

Zucco, C. Jr. (2011). "Esquerda, Direita e Governo: A Ideologia dos Partidos Políticos Brasileiros”. En T. Power y C. Zucco (Orgs,), O Congresso Por ele mesmo: Autopercepção da classe política brasileira. Belo Horizonte: Ed. UFMG. 